\title{
Transposition
}

Musique et Sciences Sociales

Hors-série 1 | 2018

Musique, histoire, sociétés

\section{Repenser la musicologie au sein des sciences humaines}

Retour sur le séminaire de François-Bernard Mâche

\section{Anne-Sylvie Barthel-Calvet}

\section{(2) OpenEdition}

\section{Journals}

Édition électronique

URL : http://journals.openedition.org/transposition/1719

DOI : 10.4000/transposition. 1719

ISSN : $2110-6134$

Éditeur

CRAL - Centre de recherche sur les arts et le langage

\section{Référence électronique}

Anne-Sylvie Barthel-Calvet, "Repenser la musicologie au sein des sciences humaines », Transposition [En ligne], Hors-série 1 | 2018, mis en ligne le 30 janvier 2018, consulté le 10 février 2020. URL : http:// journals.openedition.org/transposition/1719; DOI : 10.4000/transposition.1719

Ce document a été généré automatiquement le 10 février 2020.

La revue Transposition est mise à disposition selon les termes de la Licence Creative Commons Attribution - Partage dans les Mêmes Conditions 4.0 International. 


\title{
Repenser la musicologie au sein des sciences humaines
}

\author{
Retour sur le séminaire de François-Bernard Mâche
}

Anne-Sylvie Barthel-Calvet

À ceux qui traditionnellement mettent en doute
la musicologie, proposons une réponse précise :
nous musicologues cherchons par tous les
moyens à comprendre comment et pourquoi
l'homme a toujours et partout estimé si
important de créer des formes symboliques à
l'aide de formes sonores. (François-Bernard
Mâche, «Éclectisme et synthèse. Les conditions
d'une nouvelles musicologie générale», 1994')

1 Au seuil de son activité d'enseignement et de direction de recherche à l'EHESS, la conférence «Les universaux en musique et musicologie » prononcée en février 1994 lors du colloque La musique au regard des sciences humaines et des sciences sociales, affirme très clairement quelle est, pour François-Bernard Mâche, la place possible et légitime de la musicologie par rapport aux disciplines avec lesquelles elle entre en dialogue. Son texte sonne à la fois comme le manifeste esthétique d'un créateur et comme un plaidoyer pour une épistémologie intrinsèque à une musicologie dont les outils ne dépendent plus des autres sciences humaines mais qui soit susceptible de leur apporter l'éclairage d'un système de pensée autonome et de s'affirmer ainsi comme une interlocutrice légitime. Chez Mâche, cette réflexion épistémologique est nourrie par une pratique de création, et cette pratique de création est elle-même irriguée par une érudition que l'on aimerait qualifier de gourmande et toujours en éveil, de sorte qu'il paraît non pertinent de distinguer son activité créatrice de son activité savante. Cette érudition n'est pas celle d'un regard surplombant, mais celle d'un «homme-aumonde» qui en scrute les beautés naturelles et culturelles. Il renvoie d'ailleurs l'opposition entre nature et culture à sa banalité en montrant que la culture est souvent à l'œuvre, quoique parfois de manière souterraine, là où l'on ne la soupçonne pas et 
que, par sa présence au Monde, l'Homme instaure une irrévocable lecture culturelle, même de phénomènes purement naturels, comme le bruit de l'eau par exemple.

Dans son analyse des pratiques et patrimoines musicaux, François-Bernard Mâche rompt avec la tradition épistémologique des musicologies occidentales et propose une approche résolument et consciemment an-historique - et non anti-historique - dans une discipline placée dès sa naissance sous l'égide de l'histoire et liée à ses modes de questionnement. Rappelons que, en France, la musicologie apparaît institutionnellement, en particulier sous l'impulsion de Romain Rolland ${ }^{2}$, comme "histoire de la musique ", conçue comme une branche de l'histoire de l'art, et fait l'objet, dans l'aire germanique, d'une description catégorielle initiale qui distingue «Historische Musikwissenschaft» et "Systematische Musikwissenschaft »3. Par la suite, surtout à partir de la deuxième moitié du $\mathrm{xx}^{\mathrm{e}}$ siècle et - ce qui n'est pas fortuit parallèlement à une avant-garde qui se revendiquait en rupture avec l'héritage esthétique de la génération précédente, l'œuvre musicale a été plus fréquemment considérée per se, comme un système autonome dont il importait de décrire le fonctionnement interne, par rapport auquel l'impact des conditions d'émergence sociohistoriques était minoré. Le positionnement intellectuel de François-Bernard Mâche sort de ce schéma dichotomique, en particulier en mettant à jour l'existence d'universaux musicaux et la rémanence de structures dans des civilisations musicales très éloignées dans le temps ou l'espace. En ce sens, il propose ce que l'on peut décrire comme une «troisième voie» qui refuse de considérer la production musicale indépendamment d'autres pratiques culturelles et sociales et de l'inscrire dans la nécessité d'un flux unidirectionnel et inéluctable.

De manière assez paradoxale, cette démarche qui met en évidence les pratiques de création à l'œuvre dans des champs du vivant où on ne l'attendait pas et qui, ce faisant, réévalue la force du culturel, conteste l'orientation culturaliste des sciences humaines des années 80 - qui, en retour, se défiera de l'orientation proposée par Mâche. Mettant à jour dans l'imaginaire humain - et même animal - des traits rémanents, il remet à la fois en question la spécificité et l'autonomie de chaque culture et la part d'anthropocentrisme dont ces lectures culturalistes peuvent être porteuses. En démontrant par l'existence de liens profonds la cohérence de l'imaginaire musical dans ses expressions les plus diverses, François-Bernard Mâche s'inscrit dans une démarche épistémologique unifiée et se pose à contre-pied d'approches privilégiant une lecture autarcique des systèmes de pensée et de culture.

4 Ce faisant, le compositeur-musicologue propose un nouveau positionnement de la musicologie au sein des sciences humaines et sociales. S'il est parti dans les années soixante d'une pratique méthodologique empruntant à d'autres domaines (structuralisme lévi-straussien, analyse distributionnelle de Chomsky, etc.), il en est arrivé - et c'est la grande originalité de sa pensée - à une vision nouvelle présentant la musique et son logos à la fois comme lecture des pratiques culturelles et comme interrogation des lectures culturelles en faisant de la musique, par son haut pouvoir symbolique, le centre de gravité des activités culturelles et civilisationnelles. C'est ce qu'il affirme, sous la forme d'un quasi pronunciamento dans la conclusion d'une conférence ${ }^{4}$ donnée au Quatrième Congrès International sur la signification musicale consacré, comme de juste, aux universaux en musique :

À ceux qui traditionnellement mettent en doute la musicologie, proposons une réponse précise : nous musicologues cherchons par tous les moyens à comprendre 
comment et pourquoi l'homme a toujours et partout estimé si important de créer des formes symboliques à l'aide de formes sonores.

La clé de la recherche de Mâche musicologue (mais aussi de Mâche compositeur) est là. C'est ce qu'il explorera dans son enseignement, durant les deux années académiques qui vont suivre (1994-1995 et 1995-1996), enseignement consacré aux questions des universaux (et plus particulièrement la répétition) et des modèles en musique, dans une continuité assez évidente. Entrons un peu plus avant dans les problématiques abordées et la manière de les présenter. Comme dans ses ouvrages - au premier chef desquels Musique, Mythe, Nature ${ }^{5}$-, François-Bernard Mâche déploie une érudition virtuose, convoquant tout à tour mythologie, histoire des religions, ornithologie, bioacoustique, littérature française, psychologie de la perception, sciences cognitives, etc., et surtout manifeste, à son insu peut-être, un plaisir de l'érudition qui cherche l'extrême précision des références et des données. Ce plaisir est communicatif et cette multiplicité de domaines abordés stimule l'intérêt d'un auditoire non exclusivement composé de musicologues, mais comprenant aussi des doctorants en anthropologie, sociologie, etc. Et surtout, l'érudition n'est jamais gratuite! S'il détaille ${ }^{6}$ de nombreuses observations où l'animal semble répéter une conduite pour le plaisir sonore et en déduit qu'elle n'a pas seulement une fonction biologique, c'est non seulement pour affirmer l'existence d'un plaisir ludique et d'une dimension esthétique dans cette pratique animale, mais c'est surtout pour contester - de manière hautement novatrice et subversive - le fait que la séparation entre nature et culture ne correspond pas à celle entre animalité et humanité. Abordant la pratique de l'énumération ${ }^{7}$ dont il montre qu'elle est la forme la plus primitive commune à la poésie et à la musique, il en explique les différentes fonctions (magique, laudative, didactique et rhétorique), en explorant le Livre des Morts égyptien, les religions polythéistes, le christianisme et, passant par l'explication du Blason du Beau Tétin de Clément Marot, met en évidence sa dimension universelle d'incarnation sonore d'une pulsation répétitive primaire.

6 Avec la question des modèles, François-Bernard Mâche passe d'une lecture qui décrypte les palimpsestes des pratiques musicales à une explication des dynamiques créatrices à l'œuvre dans le choix et le fonctionnement des modèles. Après l'exhumation archéologique des indices (les rémanences de structures et pratiques dans différentes cultures animales et humaines), il entre dans une démarche dynamique d'hypothèses de lecture et d'explication dont on peut légitimement penser qu'elles se nourrissent de sa pratique créatrice. Il explore ainsi les utilisations du modèle sonore chez l'homme et l'animal et en propose deux fonctions, celles de modèle global et de modèle analytique, à travers lesquelles il interroge, par exemple, la notion de figuration. Si l'on peut de prime abord supposer que l'animal va plutôt recourir au modèle global, Mâche émet l'hypothèse que chez certains animaux, il existe une capacité analytique qui est mise en œuvre quand il réinvente un chant et que donc, l'analyse et la synthèse ne sont pas l'apanage de l'espèce humaine. Selon lui, il y a une telle profusion dans le modèle sonore que l'homme comme l'animal y choisit par élimination, et c'est la manière dont le choix est opéré, dont les éléments sont groupés, qui est révélatrice d'une combinaison entre une part sensorielle innée et une structuration induite par le conditionnement culturel.

7 Un tel cheminement de réflexion éclaire - et trahit peut-être - un des traits profonds de la pensée mâchienne qui, au fond, en décryptant le fonctionnement de l'imaginaire commun aux vivants, parle beaucoup du sien propre. Il met surtout en lumière le fait qu'il fait autant appel à la créativité dans sa réflexion scientifique qu'à la rationalité 
dans son activité artistique, et remet de ce fait en question - une fois encore - une catégorie admise, celle de l'opposition entre le savant et le créateur.

\section{BIBLIOGRAPHIE}

ADLER, Guido, « Umfang, Methode und Ziel der Musikwissenschaft », Vierteljahrsschrift für Musikwissenschaft 1 (1885), p. 5-20.

MÂCHE, François-Bernard Musique, Mythe, Nature ou les Dauphins d'Arion, Paris, Klincksieck, 1991 [1 $1^{\text {re }}$ éd. 1983].

MÂCHE, François-Bernard, «Éclectisme et synthèse. Les conditions d'une nouvelles musicologie générale ", MIEREANU, Costin et HASCHER, Xavier (eds), Les universaux en musique : actes du $4^{e}$ Congrès international sur la signification musicale, Paris, Publications de la Sorbonne, 1994, p. $155-161$

\section{NOTES}

1. MÂCHE, François-Bernard, "Éclectisme et synthèse. Les conditions d'une nouvelles musicologie générale », MIEREANU, Costin et HASCHER, Xavier (eds), Les universaux en musique: actes $d u 4^{e}$ Congrès international sur la signification musicale, Paris, Publications de la Sorbonne, 1994, p. 161.

2. Initiateur, avec Jules Combarieu, du premier congrès d'histoire de la musique qui s'est tenu à Paris en juillet 1900, Romain Rolland est nommé en 1904 titulaire de la chaire d'histoire de l'art de la Sorbonne en 1904, où il enseignera en fait l'histoire de la musique.

3. Voir ADLER, Guido, « Umfang, Methode und Ziel der Musikwissenschaft », Vierteljahrsschrift für Musikwissenschaft 1 (1885), p. 5-20.

4. MÂCHE, François-Bernard, «Éclectisme et synthèse, les conditions d'une nouvelles musicologie générale ", Quatrième Congrès International sur la signification musicale, Les universaux en musique, Paris, 9-13 octobre 1994.

5. MÂCHE, François-Bernard Musique, Mythe, Nature ou les Dauphins d'Arion, Paris, Klincksieck, 1991 [1 $1^{\text {re }}$ éd. 1983].

6. Cours du 22 janvier 1995.

7. Cours des 10 et 24 mars 1995. 


\section{AUTEUR}

\section{ANNE-SYLVIE BARTHEL-CALVET}

Ancienne élève de l'École Normale Supérieure, Anne-Sylvie Barthel-Calvet est Maître de Conférences en musicologie de la musique contemporaine à l'université de Lorraine. Elle est également chercheuse invitée au Labex GREAM de l'université de Strasbourg où elle est coordinatrice du groupe « Nouvelles Lutheries » et à l'IRCAM, dans l'équipe « Analyse des Pratiques musicales ». Spécialiste de Xenakis sur l'œuvre duquel elle a publié de nombreux articles, elle prépare actuellement deux livres sur ce compositeur, dont une biographie. Plus largement, ses recherches portent sur les avant-gardes musicales des $\mathrm{Xx}^{\mathrm{e}}$ et $\mathrm{xxI}^{\mathrm{e}}$ siècles qui sont abordées tant dans leurs aspects historiques et esthétiques que par l'analyse et l'étude génétique des œuvres. Ses travaux récents sont consacrés à l'historiographie critique de l'avant-garde de l'après-deuxième guerre mondiale (direction d'un collectif New Music Networks : Critical Historiographies of the Postwar Avant-garde à paraître chez Routledge) et à la diplomatie culturelle de la Guerre Froide. 